\title{
Preoperative computed tomography of the brain in non-small cell bronchogenic carcinoma
}

\author{
P Kormas, J R Bradshaw, K Jeyasingham
}

\begin{abstract}
Background Computed tomography of the brain is the most accurate diagnostic investigation for detecting intracranial tumours. A prospective study was undertaken to try to maximise the cost effectiveness of computed tomography of the brain in the preoperative evaluation of non-small cell lung cancer.

Methods All patients with non-small cell lung cancer who were free of neurological symptoms and were thought to be free of metastases from the results of routine investigations were subjected to computed tomography of the brain in the 12-24 hours immediately before surgery.

Results Of 158 such patients, five showed positive evidence of metastases, confirmed on craniotomy and excision biopsy; one of these patients was found to have a non-metastatic tumour (false positive). Five patients with a negative scan who underwent lung resection returned within 12 months with neurological defects and positive findings on further computed tomography (false negative). The predominant cell type in patients with positive and false negative scans was adenocarcinoma or adenosquamous carcinoma (7/10); the majority had nodal state $\mathbf{N}_{2}$.

Conclusions Computed tomography of the brain should be carried out if mediastinal disease is suspected or confirmed in non-small cell lung cancer before proceeding to surgery.
\end{abstract}

Lung cancer is the commonest primary source of brain metastases. ${ }^{12}$ The incidence of brain metastases in necropsy studies of patients dying from lung cancer varies from $30 \%$ to $50 \% .^{23}$ Of all the diagnostic tests for intracranial tumours computed tomography is the most accurate, its accuracy being equal to that of cerebral angiography. ${ }^{4}$ Cerebral angiography is an invasive procedure causing some morbidity and mortality. Computed tomography is reported to be far more accurate than radionuclide scanning, although this may sometimes be useful in combination when computed tomograms give equivocal findings. ${ }^{4}$

Routine preoperative staging in patients considered for surgery for bronchogenic carcinoma includes computed tomography of the brain. The relatively low pick up of positive scans in patients without neurological symptoms and the high cost of scanning the brains of all patients irrespective of symptoms have been highlighted in previous reports. ${ }^{56}$ The incidence of brain metastases detected by computed tomography when all patients with carcinoma of the lung were investigated in a regional cardiothoracic unit was approximately $6 \%$ in the late 1970 s. $^{5}$ Grant et al reported a retrospective study of patients whose non-small cell lung cancer was considered to be operable on the basis of a clinical history, examination, chest radiography, and bronchoscopy and who were subjected to computed tomography of the chest, abdomen, and brain at the same sitting. Approximately $3.5 \%$ of the patients had positive scans at all three sites - that is, brain, mediastinum, and abdomen-while $4.3 \%$ had deposits in the brain and mediastinum.

We present a prospective study in which computed tomography of the brain was carried out selectively in patients without neurological symptoms who had non-small cell lung cancer and appeared free of metastases in all other routine investigations, and who were therefore listed for surgery.

\section{Patients and methods}

All patients being investigated for lung cancer in our unit underwent a clinical examination, routine blood tests (which included liver function tests), chest radiography, pulmonary function tests, electrocardiography, bronchoscopy, and upper abdominal ultrasonography. If the patient complained of localised skeletal pain or if localised bone tenderness was elicited skeletal radiography of the area was undertaken. If the alkaline phosphatase activity was raised on routine blood testing, radionuclide scanning of bone was performed. When the chest lesion was peripheral and the mediastinum seemed to be normal on chest radiography computed tomography of the chest was not performed. If the lesion was central or the mediastinum seemed to be abnormal on routine chest radiography chest computed tomography was undertaken. Mediastinoscopy was not used routinely but was undertaken when computed tomography of the chest had shown lymph nodes exceeding $1.5 \mathrm{~cm}$ in diameter. The policy of the investigating surgical firm was not to exclude patients from thoracotomy if the ipsilateral mediastinal nodes on mediastinoscopy showed only intracapsular invasion by tumour. If, once these investigations had been completed, the patient was shown to have a 
Table 1 Histological cell types of carcinomas in 158 patients

\begin{tabular}{lc}
\hline Carcinoma cell type & No \\
\hline Squamous & 79 \\
Anaplastic squamous & 28 \\
Adenocarcinoma & 36 \\
Adenosquamous & 5 \\
Bronchoalveolar & 5 \\
Atypical & 5 \\
\hline
\end{tabular}

non-small cell carcinoma with no evidence of disease outside the ipsilateral chest computed tomography of the brain was arranged for the 12-24 hours before surgery, sometimes on the night or the evening before surgery, when the demands on the scanner were at their lowest. All scans were performed with a second generation General Electric 8800 scanner with $11.6 \mathrm{~s}$ slice time. Contrast medium ( $50 \mathrm{ml}$ iopromide, Scherring-a non-ionic iodine based solution) was injected slowly intravenously. The total scanning time was 25 minutes. All brain computed tomograms were reported by one consultant neuroradiologist (JRB).

\section{Results}

Of 258 patients with bronchogenic carcinoma assessed for surgery between 1 March 1987 and 28 February 1989,158 were deemed to be free of metastases before the brain scan. Of these, 121 were men and 32 women with an age range of 40 to 80 (mean $64 \cdot 1$ ) years. Table 1 shows the histological cell type distribution of these nonsmall cell lung cancers and table 2 the nodal state based on operative staging. Of the 158 patients, five showed a true positive computed tomogram and one a false positive scan, which was diagnosed as a glioma on excision biopsy of the intracranial lesion. Of 149 patients available for postoperative follow up, five returned within 12 months with neurological symptoms and evidence of brain metastases on a repeat computed tomogram of the brain. We referred to these as false negative results. The five patients with true positive results had an adenocarcinoma (three patients), squamous carcinoma (one), and adenosquamous carcinoma (one). Of the false negative scans, three represented adenosquamous, one squamous, and one anaplastic carcinoma.

Of 22 patients staged at surgery as having nodal state $\mathrm{N}_{2}$, three showed a true positive

Table 2 Nodal state in the 158 patients ${ }^{\star} \dagger$

\begin{tabular}{lclll}
\hline Nodal state & Overall & False negative & Positive & Total \\
\hline $\mathrm{N}_{0}$ & 108 & 2 & - & 2 \\
$\mathrm{~N}_{1}$ & 26 & - & - & - \\
$\mathrm{N}_{2}$ & 21 & 3 & 1 & 6 \\
$\mathrm{~N}_{2}^{\dagger}$ & 1 & - & 2 & 1 \\
\hline $\mathrm{N}_{\mathrm{x}+}^{+}$ & 2 & - & false positive $)$ & 6 \\
\hline
\end{tabular}

$\mathrm{N}_{0} v \mathrm{~N}_{1}=\mathrm{p}<0.001 ; \mathrm{N}_{1} v \mathrm{~N}_{2}=\mathrm{p}<0.01 ; \mathrm{N}_{0} v \mathrm{~N}_{2}=\mathrm{p}<0.0001$ (Student's $t$ test).

$\star$ Staging based on operative findings in the 155 patients who underwent thoracotomy. $\dagger$ Staging based on mediastinoscopy in one $\mathrm{N}_{2}$ patient.

†These two remaining patients who were not staged operatively had positive computed tomograms of the brain and at craniotomy the extent of the disease was found to be too great to justify thoracotomy. scan and three a false negative scan. None of the 26 patients staged at operation as $\mathrm{N}_{1}$ showed a true positive or false negative computed tomogram. None of the 108 staged at nodal state $\mathrm{N}_{0}$ showed a true positive scan, though two showed a false negative computed tomogram, both of whom had an adenosquamous carcinoma. In the group of patients with positive computed tomograms the predominant site of metastases was the frontal lobe of the brain, accounting for the lack of neurological symptoms.

\section{Discussion}

\section{LOGISTIC CONSIDERATIONS}

In the current climate of clinical budgeting attention is constantly focused on the cost of investigations carried out to assess patients' suitability for surgery. Computed tomography of the brain is increasingly used to evaluate patients with lung cancer. At which point in the systematic evaluation it is most economically used is a question that is likely to stimulate considerable controversy. If by performing less expensive and more routinely available investigations obvious metastases outside the chest can be detected, then the need for computed tomography of the brain in a particular patient could be eliminated. The cost of routinely scanning the brain of all patients with lung cancer referred to a regional thoracic centre can be enormous. If the brain is scanned at the same time as the chest and upper abdomen the scan time is prolonged by a variable amount, depending on the speed of the scanner, thereby rendering the scanner less available. In patients without neurological symptoms delaying computed tomography of the brain till the last investigation seems prudent so that patients are studied only when routine investigations have failed to pick up metastases outside the chest. Scanning may also be performed at a time of day or night when the demand for the scanner is at its lowest.

\section{PATIENT COMPLIANCE}

Patients were made aware of the possibility of the scan being positive, necessitating the need for a change of plan. Compliance in this series of patients was excellent. They were aware that if an intracranial metastasis was detected appropriate investigation and treatment were available on site in a regional neurosurgical centre in collaboration with the radiotherapy and oncology departments of a neighbouring hospital.

\section{PICK UP RATE}

Despite the small number of positive brain lesions detected in this study, certain useful data emerged. As the brain metastases were asymptomatic, it was not surprising to find that most of the metastases were located in the frontal region of the cerebral hemispheres. One deposit, however, was in the parietal lobe. In patients with an unequivocal scan intracranial exploration with biopsy was the primary procedure undertaken; subsequent surgery for the primary carcinoma in the lung was 
abandoned only in the three patients in whom the intracranial lesions were considered to be incurable.

In this study patients were defined as showing a false negative scan if they (a) had a negative scan when they were free of neurological symptoms and had non-small cell lung cancer with no evidence of distant metastases and $(b)$ underwent surgery and subsequently developed neurological symptoms three to 12 months afterwards with evidence of brain metastases in a second computed tomogram. This suggests that microscopic metastases were present in the brain before surgery but computed tomography was incapable of detecting them. As the nodal state in most of our patients with false negative results was $\mathrm{N}_{2}$, the clearance of mediastinal disease could have been incomplete and metastases in other organs, including the brain, could have occurred from residual disease or from dispersal of malignant cells at the time of surgery.

\section{NODAL STATE AND BRAIN METASTASES}

Our policy has been, and continues to be, to offer surgery to patients with nodal state $\mathrm{N}_{2}$, provided that their carcinoma is intracapsular (microscopic). Our results suggest that the $\mathrm{N}_{2}$ state has a significantly higher chance of a positive brain lesion even in patients free of neurological symptoms. Several patients with $\mathrm{N}_{2}$ disease did not, however, show evidence of brain metastases. Computed tomography of the brain could therefore be more profitably used in patients with $\mathrm{N}_{2}$ disease who would otherwise be subjected to surgery on the basis of intracapsular (microscopic) invasion only. The cost of treatment should be assessed not only in terms of the cost of investigations and surgery but also in terms of the psychological trauma to a patient who on the basis of preoperative investigations undergoes surgery but develops metastatic disease shortly afterwards; this suggests that proper preoperative investigations should have detected disease outside the chest. Immediate preoperative computed tomography of the brain seems to have cut this possibility by half while minimising the cost by scanning only those patients who would otherwise have undergone surgery.

1 Sunderesan N, Galicich JH, Beattie EJ. Surgical treatment of the brain metastasis from lung $J$ Neurosurg 1983;58:666-71.

2 Deviri E, Schachner A, Halevy A, Shalit M, Levit MJ. Carcinoma of lung with a solitary cerebral metastasis. Cancer 1983;52:1507.

3 Galluzzi S, Payne PM. Brain metastases from primary bronchial carcinoma: a statistical study of 741 necropsies. Br J Cancer 1956;10:408.

4 Lusins JO, Chayes Z, Hiroshi N. Computed tomography and radionuclide brain scanning comparison in evaluating metastatic lesions to brain. NY State J Med 1980;80: 185-9.

5 Cockburn CJ, Foote AB, McDonald A, Mathiews L. CAT brain scanning as a pre-operative screen in bronchial carcinoma [abstract]. In: Abstracts of the proceedings of the Society of Cardiothoracic and Vascular Surgeons of Great Britain and Ireland. London: Concorde Services, 1979.

6 Grant D, Edwards D, Goldstraw P. Computed tomography of brain, chest, and abdomen in the pre-operative assessment of non-small cell lung cancer. Thorax 1988;43: 883-6. 